\title{
On the $\mathcal{H}^{\infty}$ Controller Design for a Magnetic Suspension System Model
}

\author{
E. Karagül* H. Özbay ** \\ * Dept. of Electrical and Electronics Engineering, Bilkent University, \\ 06800 Ankara, Turkey, (e-mail: a_karagul@ug.bilkent.edu.tr) \\ ** Dept. of Electrical and Electronics Engineering, Bilkent University, \\ 06800 Ankara, Turkey, (e-mail: hitay@bilkent.edu.tr )
}

\begin{abstract}
This paper deals with the $\mathcal{H}^{\infty}$ optimal controller design for a magnetic suspension system model derived in Knospe and Zhu [2011], with added input/output delay. The plant is a fractional order system with time delay, i.e., the transfer function of the plant involves infinite dimensional terms including a rational function of $\sqrt{s}$ and $e^{-h s}$, where $h>0$ represents the delay. The $\mathcal{H}^{\infty}$ optimal controller is designed by using the recent formulation given in Özbay [2012] for the mixed sensitivity minimization problem for unstable infinite dimensional plants with low order weights. The effect of time delay on the achievable performance level is illustrated.
\end{abstract}

\section{INTRODUCTION}

Recently, in a series of papers Knospe and Zhu have obtained a fractional order mathematical model for a nonlaminated electromagnetic suspension system, see Zhu and Knospe [2010] and Knospe and Zhu [2011]. The present paper considers $\mathcal{H}^{\infty}$ controller design for this system where actuator and/or sensor time delays may be present. For the same plant a PI controller design has been proposed in Özbay et al. [2012]. In general, for fractional systems with time delays, stability windows can be determined by using the numerical procedure outlined in Fioravanti et al. [2011].

The plant under consideration has a transfer function in the form of a rational function of $\sqrt{s}$ followed by a time delay term $e^{-h s}$, where $h>0$ represents the delay amount. For such infinite dimensional systems a simple design method was developed in Toker and Özbay [1995] to compute $\mathcal{H}^{\infty}$ controllers. Recently in Özbay [2012] the formulae of Toker and Özbay [1995] has been simplified for the case where the sensitivity weight is low-order. In this paper mixed sensitivity minimizing controllers will be designed for the unstable fractional model developed in Zhu and Knospe [2010], Knospe and Zhu [2011] by using the method of Özbay [2012], and this will be verified by the old design procedure of Toker and Özbay [1995]

In Section 2 the plant model is defined and its special structure is analyzed. Section 3 contains a detailed discussion on the numerical steps for the computation of the $\mathcal{H}^{\infty}$ controller for the plant studied here. Concluding remarks are made in Section 4.

\section{PROBLEM DEFINITION}

This paper investigates the fractional order plant model of a non-laminated electromagnetic suspension system obtained in Knospe and Zhu [2011]. Possible delay effects due to sensor-actuator signal flows (real time data acqui- sion and transmission) are also considered; hence the plant transfer function is

$$
P(s)=\frac{e^{-h s}}{\left(\left(s^{\alpha}\right)^{5}+\left(s^{\alpha}\right)^{4}-c\right)}
$$

where $s$ is the Laplace variable, $\alpha$ is a rational number between 0 and 1 (in this particular case, $\alpha=0.5$ ) and $h>0$ is the time delay. A numerical stability test for fractional order systems with time delays can be done easily by using the method of Fioravanti et al. [2011]. For finding the locations of the poles of the system the following transformation plays a crucial role:

$$
\zeta=s^{\alpha} \text {. }
$$

With this transformation, stability region in the $\zeta$-plane is defined by

$$
|\angle \zeta|>\frac{\alpha \pi}{2}
$$

Knospe and Zhu [2011] shows that for all $c>0$ the plant has one unstable real pole and 4 stable complex poles. For example, taking $c=10$ gives the following poles in the $\zeta$-plane.

Table 1. Locations and phases of the roots of $\zeta^{5}+\zeta^{4}-10=0$

\begin{tabular}{|l|r|}
\hline Locations of the roots & phases \\
\hline$p_{1}=-1.5258+j 0.8868$ & $150^{\circ}$ \\
$p_{2}=-1.5258-j 0.8868$ & $-150^{\circ}$ \\
$p_{3}=0.3133+j 1.4680$ & $78^{\circ}$ \\
$p_{4}=0.3133-j 1.4680$ & $-78^{\circ}$ \\
$p=1.4250$ & $0^{\circ}$ \\
\hline
\end{tabular}

Therefore, the plant transfer function can be re-written as

$$
P(s)=e^{-h s} G\left(s^{\alpha}\right) \frac{1}{\left(s^{\alpha}-p\right)}
$$

where

$$
G\left(s^{\alpha}\right)=\frac{1}{\left(s^{\alpha}-p_{1}\right)\left(s^{\alpha}-p_{2}\right)\left(s^{\alpha}-p_{3}\right)\left(s^{\alpha}-p_{4}\right)}
$$

is the stable part of the system. Bode plots of the stable part $e^{-h s} G\left(s^{\alpha}\right)$ are shown in Fig. 1 . 

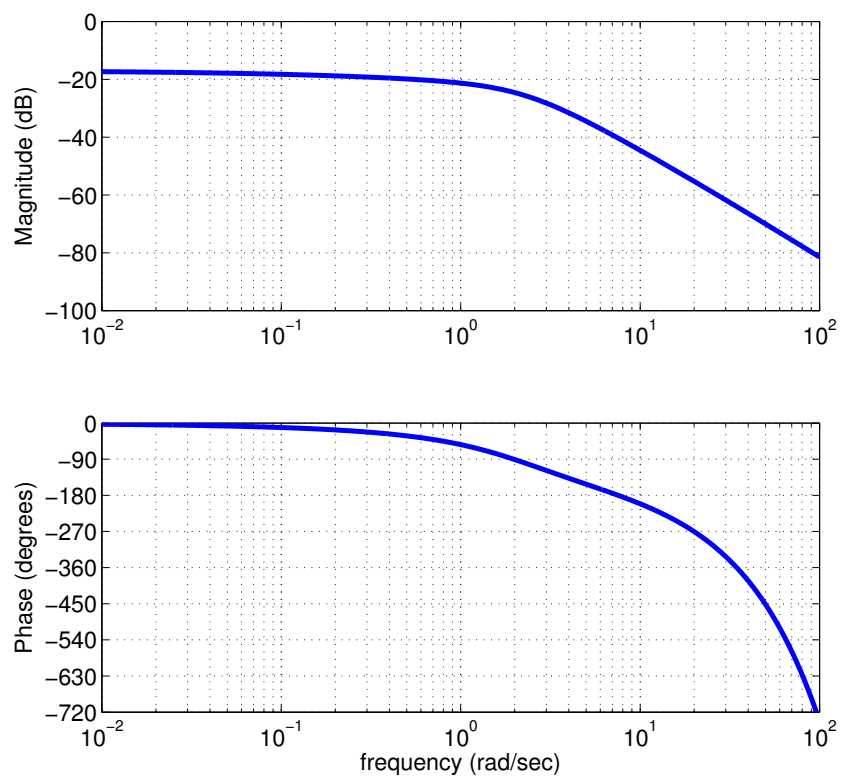

Fig. 1. Bode plots of $e^{-h s} G\left(s^{\alpha}\right)$ for $h=0.1, c=10$ and $\alpha=0.5$

\section{DESIGNING OPTIMAL $\mathcal{H}^{\infty}$ CONTROLLER}

In this section, the $\mathcal{H}^{\infty}$ controller formula given in Toker and Özbay [1995], and the new method suggested in Özbay [2012] will be applied separately to design the optimum $\mathcal{H}^{\infty}$ controller. This section is divided into three parts, first factorization of the plant will be given, then in the following two subsections, optimum performance level will be investigated by the above mentioned methods separately.

\subsection{Factorization of the Plant}

For the system model given above the mixed sensitivity minimization problem tries to find the optimum performance level and the corresponding optimal controller:

$$
\begin{aligned}
\gamma_{o p t}: & =\min _{C \in \mathcal{C}(P)}\left\|\left[\begin{array}{c}
W_{1}(1+P C)^{-1} \\
W_{2} P C(1+P C)^{-1}
\end{array}\right]\right\|_{\infty} \\
& =\left\|\left[\begin{array}{c}
W_{1}\left(1+P C_{o p t}\right)^{-1} \\
W_{2} P C_{o p t}\left(1+P C_{o p t}\right)^{-1}
\end{array}\right]\right\|_{\infty} .
\end{aligned}
$$

In $(2), \mathcal{C}(P)$ denotes the set of all of controllers stabilizing the closed loop feedback system with the plant $P$; the filters $W_{1}(s)$ and $W_{2}(s)$ are rational weighting functions shaping the desired sensitivity and the complementary sensitivity, respectively. Recall that $C \in \mathcal{C}(P)$ if and only if $(1+P C)^{-1}, C(1+P C)^{-1}$ and $P(1+P C)^{-1}$ are in $\mathcal{H}_{\infty}$. Typically, $W_{1}(s)$ is a low order, low pass filter representing a reference signal generator and $W_{2}(s)$ is a high pass filter representing an upper bound on the multiplicative uncertainty of the plant. The plant in (1) can be written in the form

$$
P(s)=\frac{M_{n}(s) N_{o}(s)}{M_{d}(s)} .
$$

where $N_{o}(s)$ is an outer function, $M_{n}(s)$ is an inner function, and $M_{d}(s)$ is a rational inner function whose zeroes $\alpha_{1}, \ldots, \alpha_{l} \in \mathbb{C}_{+}$are the unstable poles of the system.
The formula in Toker and Özbay [1995] requires $M_{d}(s)$ to be the rational function of $s$. To put the plant into the framework of (3) we take advantage of the fact that $\alpha=0.5$ and hence $\left(s^{\alpha}-p\right)\left(s^{\alpha}+p\right)=\left(s-p^{2}\right)$ :

$$
\begin{gathered}
M_{n}(s)=e^{-h s} \\
M_{d}(s)=\frac{\left(s-p^{2}\right)}{\left(s+p^{2}\right)} \\
N_{o}(s)=\frac{\left(s^{\alpha}+p\right)}{\left(s+p^{2}\right)\left(s^{\alpha}-p_{1}\right)\left(s^{\alpha}-p_{2}\right)\left(s^{\alpha}-p_{3}\right)\left(s^{\alpha}-p_{4}\right)}
\end{gathered}
$$

Thus, in the specific example considered here $l=1$ and $\alpha_{1}=p^{2}$. In this study, for simplicity of the exposition low order weights are chosen:

$$
W_{1}(s)=\frac{1}{s} \quad W_{2}(s)=k s \quad k=0.3
$$

and the notation $W_{1}=n W_{1} / d W_{1}$ is used with $n W_{1}(s)=1$ and $d W_{1}(s)=s$.

\subsection{Toker-Özbay Formula}

For the factorized plant, (3), the $\mathcal{H}^{\infty}$ controller can be written in the form

$$
C=E_{\gamma} M_{d} \frac{N^{-1} F_{\gamma} L}{1+M_{n} F_{\gamma} L}
$$

where

$$
\begin{aligned}
& E_{\gamma}(s)=\frac{W_{1}(-s) W_{1}(s)}{\gamma^{2}}-1 \\
& F_{\gamma}(s)=\frac{d W_{1}(-s)}{n W_{1}(s)} \gamma G_{\gamma}(s) .
\end{aligned}
$$

The stable function $G_{\gamma}(s)$ is obtained from the spectral factorization:

$G_{\gamma}(s) G_{\gamma}(-s)=\left(1+\frac{W_{2}(-s) W_{2}(s)}{W_{1}(-s) W_{1}(s)}-\frac{W_{2}(-s) W_{2}(s)}{\gamma^{2}}\right)^{-1}$.

The controller, (4), will achieve the optimum level performance if we put $\gamma=\gamma_{o p t}$ and find the corresponding $L(s)$. For finding these two missing items, $\gamma_{o p t}$ and $L(s)$, the following set of computations are performed. First, define

$$
L(s)=\frac{\left[1 s \ldots s^{n-1}\right] \Psi_{2}}{\left[1 s \ldots s^{n-1}\right] \Psi_{1}}
$$

where $n:=n_{1}+l$, with $n_{1}=\operatorname{deg}\left(d W_{1}\right)$. The unknown coefficients $\Psi_{1}$ and $\Psi_{2}$ are defined in the following way: $\Psi_{1}=\left[\begin{array}{lll}\psi_{10} & \ldots & \psi_{1(n-1)}\end{array}\right]^{T}, \Psi_{2}=\left[\begin{array}{llll}\psi_{20} & \ldots & \psi_{2(n-1)}\end{array}\right]^{T}$. The relationship between $\Psi_{1}$ and $\Psi_{2}$ is

$$
\Psi_{1}= \pm \mathscr{J}_{n} \Psi_{2}, \mathscr{J}_{n} \Psi_{2}=: \Phi,
$$

where $\mathscr{J}_{n}$ is $n \times n$ diagonal matrix, whose $i^{t h}$ diagonal entry is equal to $(-1)^{i+1}$. The function $L(s)$ is determined from $\Phi$, the singular vector of $\mathscr{R}_{\gamma}$ corresponding to zero singular value obtained by the largest feasible $\gamma>0$ :

$$
\mathscr{R}_{\gamma} \Phi=0
$$

where the parameterized matrix $\mathscr{R}_{\gamma}$ is given by

$$
\mathscr{R}_{\gamma}=\left[\begin{array}{cc}
\mathscr{V}_{\alpha}^{l} & \mathscr{D}_{\alpha} \mathscr{V}_{\alpha}^{n_{1}} \\
\mathscr{V}_{\beta}{ }^{l} & \mathscr{D}_{\beta} \mathscr{V}_{\beta}^{n_{1}}
\end{array}\right] \pm\left[\begin{array}{cc}
\mathscr{D}_{l} & 0 \\
0 & \mathscr{D}_{n_{1}}
\end{array}\right]\left[\begin{array}{cc}
\mathscr{V}_{\alpha}{ }^{l} & \mathscr{D}_{\alpha} \mathscr{V}_{\alpha}^{n_{1}} \\
\mathscr{V}_{\beta}{ }^{l} & \mathscr{D}_{\beta} \mathscr{V}_{\beta}{ }^{n_{1}}
\end{array}\right] \mathscr{J}_{n}
$$

The above definition uses the following:

$$
\begin{gathered}
\mathscr{D}_{l}=\operatorname{diag}\left(M_{n}\left(\alpha_{1}\right) F_{\gamma}\left(\alpha_{1}\right), \ldots, M_{n}\left(\alpha_{l}\right) F_{\gamma}\left(\alpha_{l}\right)\right) \\
\mathscr{D}_{n_{1}}=\operatorname{diag}\left(M_{n}\left(\beta_{1}\right) F_{\gamma}\left(\beta_{1}\right), \ldots, M_{n}\left(\beta_{n_{1}}\right) F_{\gamma}\left(\beta_{n_{1}}\right)\right)
\end{gathered}
$$




$$
\mathscr{D}_{n}=\operatorname{blockdiag}\left(\mathscr{D}_{l}, \mathscr{D}_{n_{1}}\right)
$$

$\mathscr{V}_{x}{ }^{m}$ denotes $k \times m$ dimensional Vandermonde matrix, constructed from a given vector $x=\left[\begin{array}{ll}x_{1} & \ldots \\ x_{k}\end{array}\right]^{T} \in \mathbb{C}^{k}$ and $\beta_{1}, \ldots, \beta_{n 1} \in \mathbb{C}_{+}$are the zeros of $E_{\gamma}(s)$. With the above equations, it is possible to obtain the parameterized matrix, $\mathscr{R}_{\gamma}$. This will be used to find $\gamma_{o p t}$ and $L(s)$. The optimal performance level $\gamma_{o p t}$ is the largest value of $\gamma$ which makes $\mathscr{R}_{\gamma}$ singular. After finding $\gamma_{o p t}$ and the value of $\mathscr{R}_{\gamma}, \Psi_{2}$ and $\Psi_{1}$ can be found by (6).

\subsection{Simplified Method Given by Özbay [2012]}

In the previous subsection, to reach optimum performance a parameterized matrix is used. On the other hand, as shown in Özbay [2012], when $W_{1}(s)$ is first order, (7) can be reduced to a scalar equation $\mathscr{P}_{\gamma}=0$, where

$$
\begin{gathered}
\mathscr{P}_{\gamma}=b\left(I \pm M_{n}\left(\beta_{1}\right) F_{\gamma}\left(\beta_{1}\right) \mathscr{J}_{l}\right)\left(I \pm M_{n}\left(A_{d}\right) F_{\gamma}\left(A_{d}\right) \mathscr{J}_{l}\right)^{-1} \\
\left(I \pm M_{n}\left(A_{d}\right) F_{\gamma}\left(A_{d}\right)(-1)^{l}\right) a+\left(I \pm M_{n}\left(\beta_{1}\right) F_{\gamma}\left(\beta_{1}\right)(-1)^{l}\right),
\end{gathered}
$$

with

$$
\begin{gathered}
A_{d}=\left[\begin{array}{cccc}
0 & \cdots & 0 & -a_{0} \\
1 & \cdots & 0 & -a_{1} \\
\ddots & & \vdots \\
0 & 1 & -a_{l}
\end{array}\right] \\
a=-\left[a_{0}, a_{1}, \ldots, a_{l-1}\right]^{T} \\
b=-\beta_{1}{ }^{-l}\left[1, \beta_{1}, \ldots, \beta_{1}^{l-1} .\right]
\end{gathered}
$$

Since $M_{d}(s)$ is first order $l=1$ and $A_{d}=-a_{o}=p^{2}$. Also, since $W_{1}(s)$ is first order, $b=-1 / \beta_{1}$ and $\beta_{1}=j / \gamma$. The largest $\gamma$ value making $\mathscr{R}_{\gamma}$ singular is $\gamma_{\text {opt }}$, and this is also the largest $\gamma$ satisfying $\mathscr{P}_{\gamma}=0$. Therefore, both (7) and (9) can be used to find $\gamma_{o p t}$. Fig. 2 illustrates this point for some particular choices of $h$ and $c$.

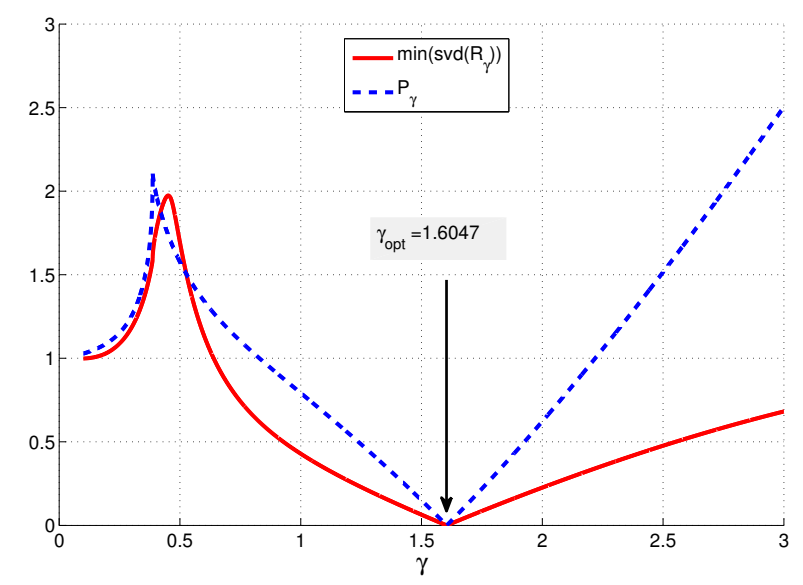

Fig. 2. $\gamma$ vs. $\min \left(\operatorname{svd}\left(\mathscr{R}_{\gamma}\right)\right)$ (solid line) and $\mathscr{P}_{\gamma}$ (dashed line); consistency is verified, $\gamma_{\text {opt }}=1.6047$ for $h=0.1$ and $c=10$

As seen from Fig. 2, $\gamma_{\text {opt }}=1.6047$ value computed from two different methods coincide. Corresponding first order function $L(s)$ can be computed as summarized above and hence the optimal controller can be constructed from

$$
C_{o p t}=E_{\gamma_{o p t}} M_{d} \frac{N_{o}^{-1} F_{\gamma_{o p t}} L}{1+M_{n} F_{\gamma_{o p t}} L} .
$$

\subsection{Optimal Controller}

Once $\gamma_{\text {opt }}$ is computed as above, corresponding $\mathscr{R}_{\gamma}$ is determined as

$$
\mathscr{R}_{\gamma}=\left[\begin{array}{cc}
1.7067 & 0.5956 \\
1.5226+j 0.85 & 0.5313+j 0.3
\end{array}\right]
$$

whose singular vector gives

$$
\Psi_{2}=\left[\begin{array}{ll}
-0.3295 & -0.9442
\end{array}\right]
$$

that leads to

$$
L(s)=\frac{0.9442 s+0.3295}{0.9442 s-0.3295}
$$

Now with the $\gamma_{\text {opt }}$ value computed, numerical values of the functions $E_{\gamma_{o p t}}(s)$ and $F_{\gamma_{o p t}}(s)$ can be obtained:

$$
\begin{gathered}
E_{\gamma_{o p t}}(s)=\frac{1+\gamma_{o p t} s^{2}}{-\gamma_{o p t} s^{2}} \\
F_{\gamma_{o p t}}(s)=\frac{-\gamma_{o p t} s}{k s^{2}+k_{a} s+1} ; \quad \text { where } \quad k_{a}=0.7517 .
\end{gathered}
$$

Now, with the above functions determined, the controller defined by (9) can be constructed and its frequency response plots can be easily obtained. In order to illustrate the effect of time delay on the optimal controller, Bode diagrams of $C_{\text {opt }}(s)$ for two different values of $h(h=0.1$ and $h=0.2$ ) are given in Fig. 3 .

Also, in Fig. 4 the effect of time delay on the achievable performance level is shown. Note that $\gamma_{o p t}$ increases exponentially with $h$.
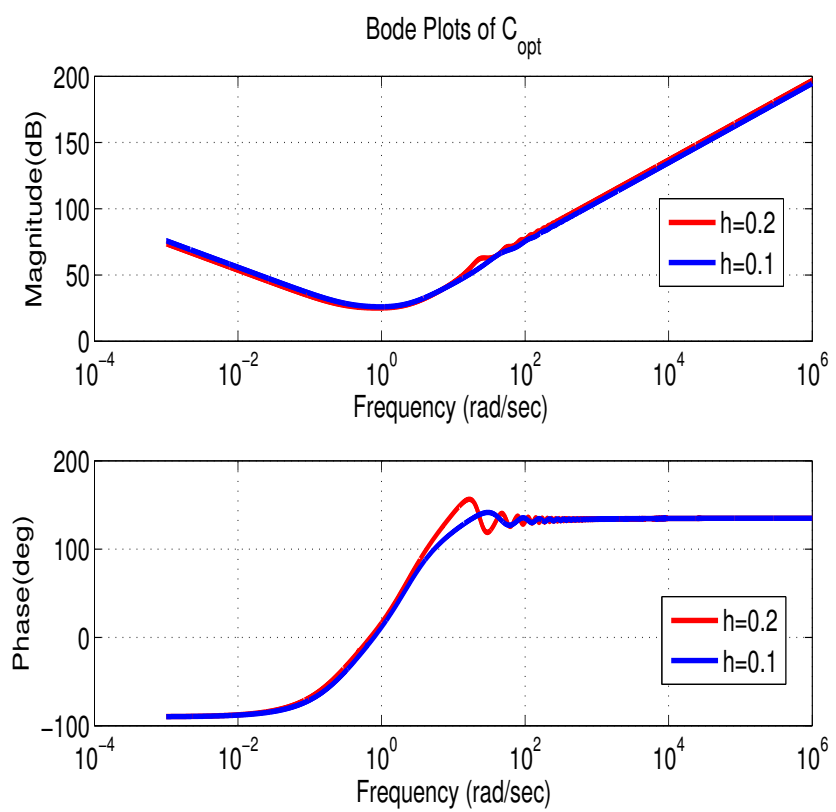

Fig. 3. Magnitude and Phase Diagrams of $C_{o p t}$

As seen from Fig. 3 the optimal controller is improper. A suboptimal proper controller is desired for many obvious reasons. For this purpose, a low pass filter in the form $1 /(\epsilon s+1)^{v}$ is connected in series with the optimal controller. In the low pass filter, $v$ is defined to be 2 so that controller is strictly proper, and $\epsilon$ is defined to be 0.005 to create a roll off in in the magnitude plot of $\left|C_{\text {subopt }}(j \omega)\right|=\left|C_{\text {opt }}(j \omega) /(1+j \epsilon \omega)^{v}\right|$ for $\omega \geq 200 \mathrm{rad} / \mathrm{sec}$. 


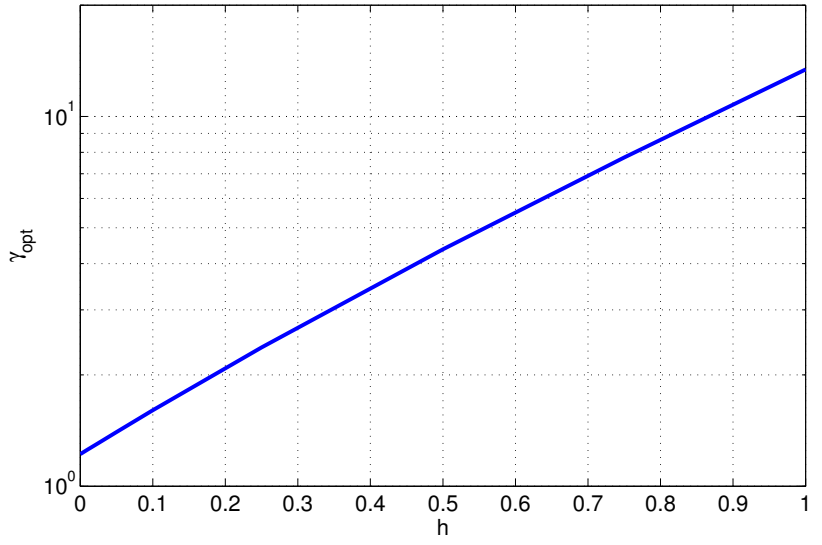

Fig. 4. Performance level $\gamma_{o p t}$ versus time delay $(c=10)$.
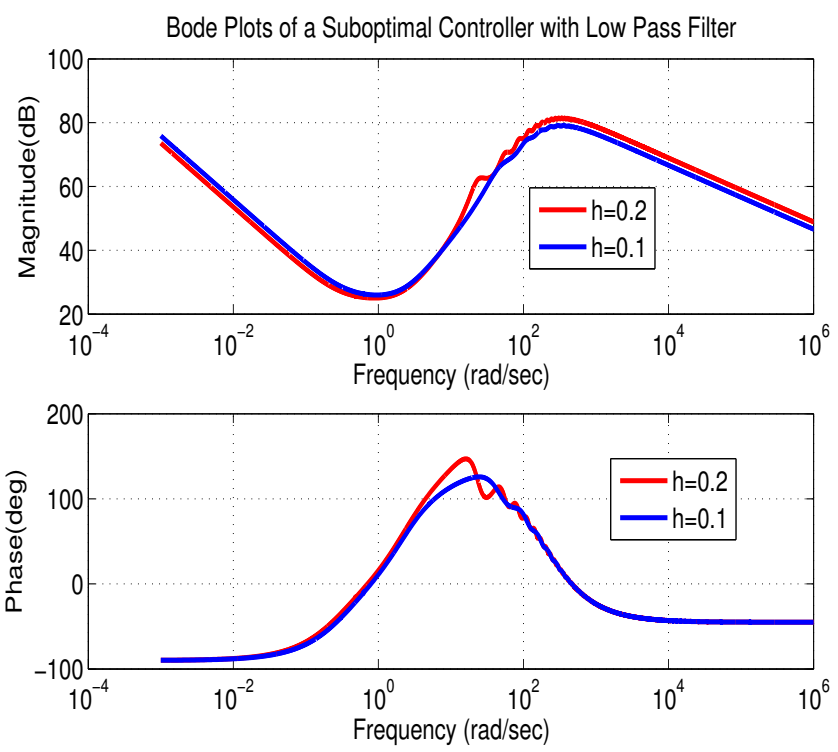

Fig. 5. Suboptimal Controller with Low Pass Filter.

By simple computations similar to the ones illustrated in Fig. 7, it can be shown that the relationship between $\gamma_{o p t}$ and $\gamma\left(C_{\text {subopt }}\right)$ (actual performance level of the suboptimal controller) satisfies $\gamma_{o p t} \leq \gamma\left(C_{\text {subopt }}\right) \leq 1.01 \gamma_{o p t}$ for all $h \leq 0.2 \mathrm{sec}$ and $\epsilon \leq 0.005$.

Fig. 6 illustrates the weighted sensitivity and complementary sensitivity $W_{1} S$ and $W_{2} T$ corresponding to $C_{\text {subopt }}$ for $h=0.1$ and $h=0.2$.

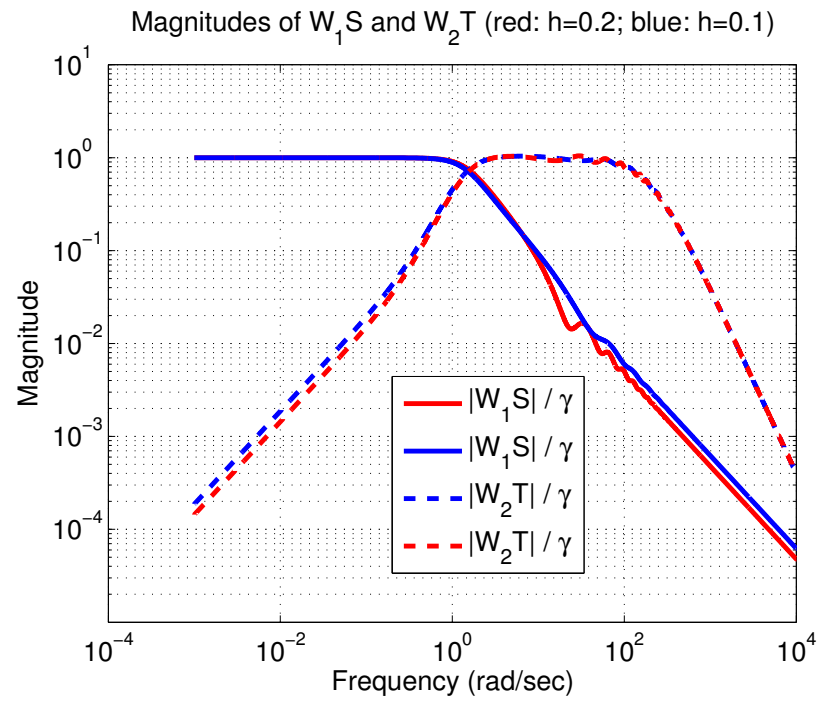

Fig. 6. $\left|W_{1} S / \gamma\right|$ and $\left|W_{2} T / \gamma\right|$ for $h=0.2$ and $h=0.1$.

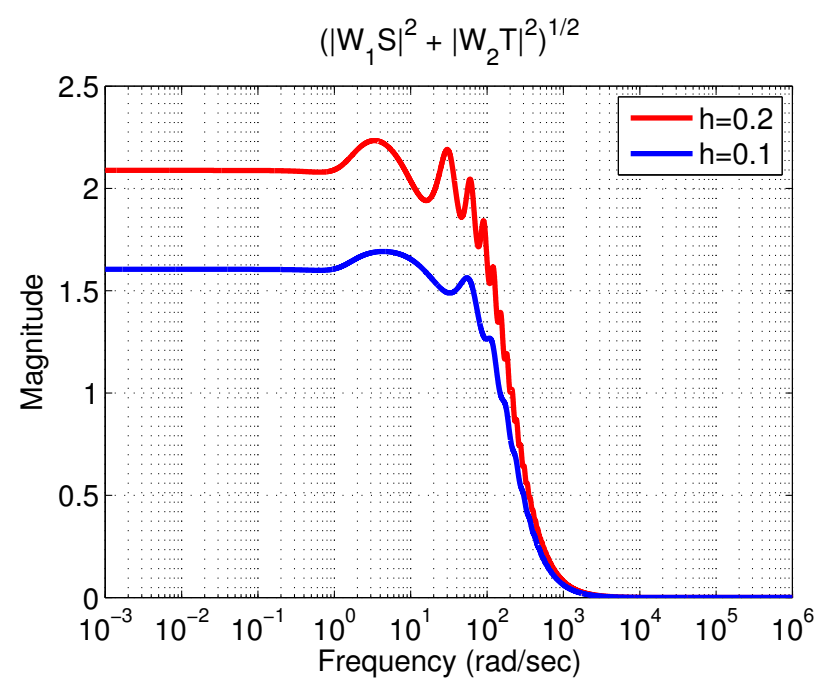

Fig. 7. $\sqrt{\left|W_{1} S\right|^{2}+\left|W_{2} T\right|^{2}}$ for $h=0.2$ and $h=0.1$.

\section{CONCLUSIONS}

In this paper, $\mathcal{H}^{\infty}$ optimal controller is computed for a fractional order model of a non-laminated magnetic suspension system with time delay. In this design, a recently developed computational method given in Özbay [2012] is verified with the earlier proven technique of Toker and Özbay [1995]. The weighting functions are chosen arbitrarily. Since $W_{2}(s)$ is rational and $G\left(s^{\alpha}\right)$ is fractional order, Bode magnitude plot of $C_{\text {opt }}$ shows a fractional order improper behavior as $s=j \omega, \omega \rightarrow \infty$. In order to avoid such an undesirable behavior, a suboptimal controller is obtained by adding a low pass filter whose cut-off frequency is around $200 \mathrm{rad} / \mathrm{sec}$.

As future work, time domain responses of the fractional order system with the computed $\mathcal{H}^{\infty}$ controller are to be studied. 


\section{REFERENCES}

A. R. Fioravanti, C. Bonnet, H. Özbay and S-I. Niculescu. Stability windows and unstable root loci for linear fractional order systems The 18th IFAC World Congress, Milan, Italy, pp. 12532-12537, 2011.

C. R. Knospe and L. Zhu. Performance limitations of nonlaminated magnetic suspension systems. IEEE Trans. on Control Systems Technology, Vol. 19, No. 2, pp. 327336, 2011.

H. Özbay. Computation of $\mathcal{H}^{\infty}$ controllers for infinite dimensional plants using numerical linear algebra. Numer. Linear Algebra Appl, doi: 10.1002/nla.1809, 2012.

H. Özbay, C. Bonnet and A. R. Fioravanti, PID controller design for fractional-order systems with time delays. Systems \& Control Letters, vol. 61 pp. 18-23, 2012.

O. Toker and H. Özbay. $\mathcal{H}^{\infty}$ optimal and suboptimal controllers for infinite dimensional SISO plants IEEE Trans. on Automatic Control, Vol. 40, No. 4, pp. 751$755,1995$.

L. Zhu and C. R. Knospe. Modeling of Nonlaminated Electromagnetic Suspension Systems. IEEE Trans. on Mechatronics, Vol. 15, No. 1, pp. 59-69, 2010. 